\title{
Synthesis of $\mathrm{SiC}$ nanowhiskers from graphite and silica by microwave heating
}

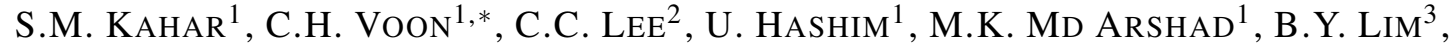 \\ S.C.B. GOPINATH ${ }^{1,4}$, W. RAHMAN ${ }^{2}$ \\ ${ }^{1}$ Institute of NanoElectronic Engineering, Universiti Malaysia Perlis, Seriab, 01000, Kangar, Perlis, Malaysia \\ ${ }^{2}$ School of Manufacturing Engineering, Universiti Malaysia Perlis Kampus Alam Pauh Putra, 02600, Arau, Perlis, Malaysia \\ ${ }^{3}$ School of Materials Engineering, Universiti Malaysia Perlis, Jejawi, Arau, 02600 Perlis, Malaysia \\ ${ }^{4}$ School of Bioprocess Engineering, Universiti Malaysia Perlis, 02600 Arau, Perlis, Malaysia
}

\begin{abstract}
Silicon carbide ( $\mathrm{SiC}$ ) is an important ceramics for engineering and industrial applications due to its advantage to withstand in high temperatures. In this article, a demonstration of $\mathrm{SiC}$ nanowhiskers synthesis by using microwave heating has been shown. The mixtures of raw materials in the form of pellets were heated, using a laboratory microwave furnace, to $1400{ }^{\circ} \mathrm{C}$ for 40 minutes at a heating rate of $20{ }^{\circ} \mathrm{C} / \mathrm{min}$. The characterization process proved that the mixture of graphite and silica in the ratio of 1:3 is an ideal composition for synthesizing single phase $\beta$-SiC nanowhiskers. Vapor-solid mechanism was suggested to explain the formation of $\mathrm{SiC}$ nanowhiskers by the proposed microwave heating.
\end{abstract}

Keywords: microwave heating; silicon carbide nanowhiskers; graphite; silica

(C) Wroclaw University of Technology.

\section{Introduction}

Silicon carbide $(\mathrm{SiC})$ is one of the most popular ceramics used in the industry. It has unique characteristics such as high melting point, excellent oxidation resistance, high chemical inertness, high thermal conductivity, good microwave absorbing ability, wide energy band gap and high mechanical strength enabling $\mathrm{SiC}$ to be used widely in aerospace structures, biomaterials and high temperature semiconducting devices [1-6]. SiC is produced mainly in industry by Acheson process. This process named after its inventor Edward Goodrich Acheson produces $\mathrm{SiC}$ by heating the mixture of quartz sand and powdered coke (carbon) in an iron bowl using voltages of $40,000 \mathrm{~V}$ to $50,000 \mathrm{~V}$ for 20 hours. Obviously, this process consumes much energy and time. Subsequently, studies have been conducted to overcome this issue. Different methods have been proposed to produce SiC. These methods include physical evaporation $[7,8]$,

\footnotetext{
*E-mail: chvoon@unimap.edu.my
}

carbon thermal reduction [9], sol gel process [10, 11] and chemical vapor deposition [12, 13]. Although these processes can successfully synthesize $\mathrm{SiC}$, there are still some drawbacks that limit the introduction of these methods to industrial processes, such as high energy consumption, long processing time and extensive chemicals usage. Other than that processes produce $\mathrm{SiC}$ with impurities at the end of reaction [7].

Lately, researchers have implemented microwave heating to synthesize inorganic materials [14-17]. Microwaves can volumetrically heat materials and give sudden increase in the temperature of a material, comparing to conventional heating processes that rely on external radiant energy to heat materials by conduction, convection and radiation. By using microwave processing, reaction rate can be increased significantly compared to conventional heating which enables the synthesis of $\mathrm{SiC}$ in large quantities [14]. Another researcher, Mingos [15] proposed that synthesis of inorganic material using microwave heating can enhance mechanical properties of the material. The rapid heating 
by microwaves may limit the extent of nonisothermal processes, e.g. segregation of impurities to the grain boundaries. Also, since the sintering time is generally reduced, the possibility of secondary crystallization may be reduced and thereby lead to improving the mechanical properties of the materials [16]. Silicon carbide nanowhisker ( $\mathrm{SiCNW}$ ) is a $\mathrm{SiC}$ with $1-\mathrm{D}$ nanostructure that can be observed under a microscope in a whisker/needle form. SiCNWs provide many advantages to the electronic devices such as sensors, field emitting diodes and solar cells due to the whiskers form of the material [18].

In this study, microwave heating was used to synthesize SiCNWs from a mixture of graphite and silica since it is generally faster, cleaner, and more economical than the conventional methods [17]. The effect of the ratio of silica and graphite was studied to determine the ideal proportion of silica and graphite to synthesize SiCNWs. Several studies have been done to study the effect of different ratios of silica-based and carbon-based starting material to synthesize SiC. For example, Tong et al. [19] have studied the effect of ratio of $\mathrm{CH}_{4}: \mathrm{SiO}_{2}$ on the synthesis $\mathrm{SiC}$ nanopowders by using thermal plasma synthesis. They found that the ratio of $\mathrm{CH}_{4}: \mathrm{SiO}_{2}$ has a significant effect on the purity of SiC. Zhu et al. [20] have also studied the effect of $\mathrm{C}_{2} \mathrm{H}_{4}$ and $\mathrm{SiH}_{4}$ ratio on the synthesis of $\mathrm{SiC}$ nanopowders through chemical vapor deposition. They found that single $\beta-\mathrm{SiC}$ phase can be obtained only for $\mathrm{C}_{2} \mathrm{H}_{4}$ and $\mathrm{SiH}_{4}$ in the ratio of $1: 2$. Therefore, the ratio of raw materials is believed to have significant influence on the quality and purity of the end product. To the best of our knowledge, there is no report on the effect of ratio of silica and graphite on the synthesis of SiCNWs using microwave heating. Thus, in this study, the ratio of silica and graphite is considered as an important parameter to optimize the synthesis of SiCNWs.

\section{Experimental}

\subsection{Sample preparation}

Silica (particle size $\leqslant 50 \mu \mathrm{m}$ ) and extra pure fine graphite powder (particle size $\leqslant 50 \mu \mathrm{m}$ ) were used as starting material. Mixtures of graphite and silica of different weight ratios of 1:1, 1:3, 1:5 and 1:7 with a total weight of $1 \mathrm{~g}$ were acquired. Ethanol was used as the liquid medium to mix the raw materials. Ultrasonic mixing bath was used as an external mean to generate vibrations in the ethanol for homogeneous mixing of the raw materials. The mixtures were then dried using a hot plate to vaporize the liquid medium. Before subjecting to microwave heating, the mixture was compressed into pellets. The process of making pellet is essential to separate the mixture of $\mathrm{SiO}_{2}$ and graphite from the graphite enveloping the pellet inside the crucible. The pressure applied to the mixture during the compression process was $312.2 \mathrm{MPa}$ to ensure full compression of the mixtures.

\subsection{Synthesis of SiCNWs by microwave heating}

Microwave heating was performed in a Synotherm microwave sintering furnace, model MW-L0316V with a multimode cavity in which $2.45 \mathrm{GHz}$ microwave irradiation was brought out through a waveguide.

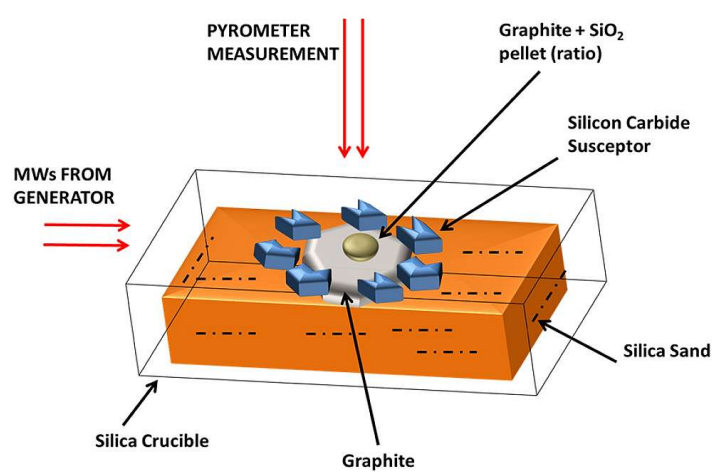

Fig. 1. Setup for sample preparation inside the microwave cavity.

The pellet was placed in a silica crucible and the crucible was placed in the microwave cavity as shown in Fig. 1. Silica sand was used as a heat insulator to prevent heat loss. SiC susceptor functioned as microwave absorber to absorb and convert electromagnetic energy to heat because $\mathrm{SiC}$ susceptor is a good microwave absorber material. 
The pellets were heated to $1400{ }^{\circ} \mathrm{C}$ with a heating rate of $20^{\circ} \mathrm{C} / \mathrm{min}$ and soaked for 40 minutes.

\subsection{Characterization of SiCNWs}

After the microwave heating was done, the samples were characterized using X-ray diffraction (XRD), field emission scanning electron microscopy (FESEM), energy-dispersive X-ray spectroscopy (EDX), photoluminescence spectroscopy (PL), Fourier transform infrared spectroscopy (FTIR) and thermo-gravimetric analysis (TGA).

The morphologies of the samples were observed by using FESEM model Nova Nano 450 at magnification of $200,000 \times$ and accelerating voltage of $5 \mathrm{kV}$ while EDX (EDX OXFORD FM29142) was used to determine the elemental composition of the specimens. Meanwhile, XRD Siemens diffractometer, model $\mathrm{D}-5000$ using $\mathrm{CuK} \alpha$ radiation source in $\theta / 2 \theta$ mode was used to investigate the composition of the specimens. The measurements were made with fast duration scan $(1 \mathrm{~s})$ and small step size $\left(0.02^{\circ}\right)$. Optical properties of SiCNWs synthesized from the mixtures were identified by using the photoluminescence spectroscopy (PL FL3-11 J81040) with a xenon lamp at $400 \mathrm{~W}$ and excitation wavelength of $360 \mathrm{~nm}$ and recorded in the wavelength range of $300 \mathrm{~nm}$ to $650 \mathrm{~nm}$. FT-IR MAGNA550 $\mathrm{kBr}$ was used to scan the samples from $500 \mathrm{~nm}^{-1}$ to $4000 \mathrm{~nm}^{-1}$ with the spectral resolution of $4 \mathrm{~nm}^{-1}$. Quality of SiCNWs was evaluated indirectly by using PerkinElmer Pyris 6 TGA analyzer. Samples about $10 \mathrm{mg}$ weight were heated from $30{ }^{\circ} \mathrm{C}$ to $1300{ }^{\circ} \mathrm{C}$ with a heating rate of $10{ }^{\circ} \mathrm{C} / \mathrm{min}$ in atmospheric air to investigate the quality of the synthesized SiCNWs.

\section{Result and discussion}

\subsection{Characterization of SiCNWs using FESEM}

Fig. 2 shows the FESEM images of samples with different ratios of $\mathrm{SiO}_{2}$ and graphite, subjected to microwave heating. It can be seen that the ratio of $\mathrm{SiO}_{2}$ and graphite, significantly influences the synthesis of SiCNWs. Fig. 2a shows the mixture of $\mathrm{SiO}_{2}$ and graphite in the 1:1 ratio after subjecting to microwave heating. It can be observed that only a little amount of nanowhiskers (blue circles) were formed and the particles in microsize (orange circle) are unreacted $\mathrm{SiO}_{2}$. It is believed that this is due to the fact that the amount of graphite was not sufficient to react with the $\mathrm{SiO}_{2}$. Similar observation was reported by Biernacki et al. [21]. They reported that in case of insufficient amount of carbon, the formed $\mathrm{SiC}$ may react with excess silica to form $\mathrm{SiO}$ and $\mathrm{CO}$ gases at high temperatures causing the loss of SiC. Thus, $\mathrm{SiC}$ nanowhiskers were not formed or formed in too small amount to be observed due to the reaction between the formed $\mathrm{SiC}$ with excess $\mathrm{SiO}_{2}$ based on the following reaction:

$$
2 \mathrm{SiO}_{2}(\mathrm{~g})+\mathrm{SiC}(\mathrm{s})+3 \mathrm{SiO}(\mathrm{g})+\mathrm{CO}(\mathrm{g})
$$

Fig. 2b shows the FESEM images of SiCNWs formed from the mixture of $\mathrm{SiO}_{2}$ and graphite in the ratio of $1: 3$ that was subjected to microwave heating. $\mathrm{SiC}$ in the form of nanowhiskers can be clearly observed. The diameters of the SiCNWs are uniform along the length of the nanowhiskers. No particles of graphite or silica are observed, which means that all graphite and silica were converted to SiCNWs. The ratio of 1:3 of silica and graphite is believed to be the ideal for synthesis of SiCNWs. Li et al. [10] also reported similar result for $\mathrm{SiC}$ in the form of nanowhiskers without a trace of raw materials, obtained using silica sol and phenolic resin (carbon) in the ratio of 1:3.

Fig. 2c shows the SiCNWs formed by microwave heating of a mixture of $\mathrm{SiO}_{2}$ and graphite in the ratio of 1:5. It can be observed that the amount $\mathrm{SiC}$ nanowhiskers (blue circle) is less than those formed in Fig. 2b. Irregular particles can be observed (red circle). The residue left in the product might be excess unreacted graphite. Similar observation was made for SiCNWs formed by microwave heating $\mathrm{SiO}_{2}$ and graphite mixture in the ratio of 1:7. The amount of unreacted graphite particles is higher. It is believed that the presence of unreacted graphite is due to the insufficient amount of silica in the starting material. 


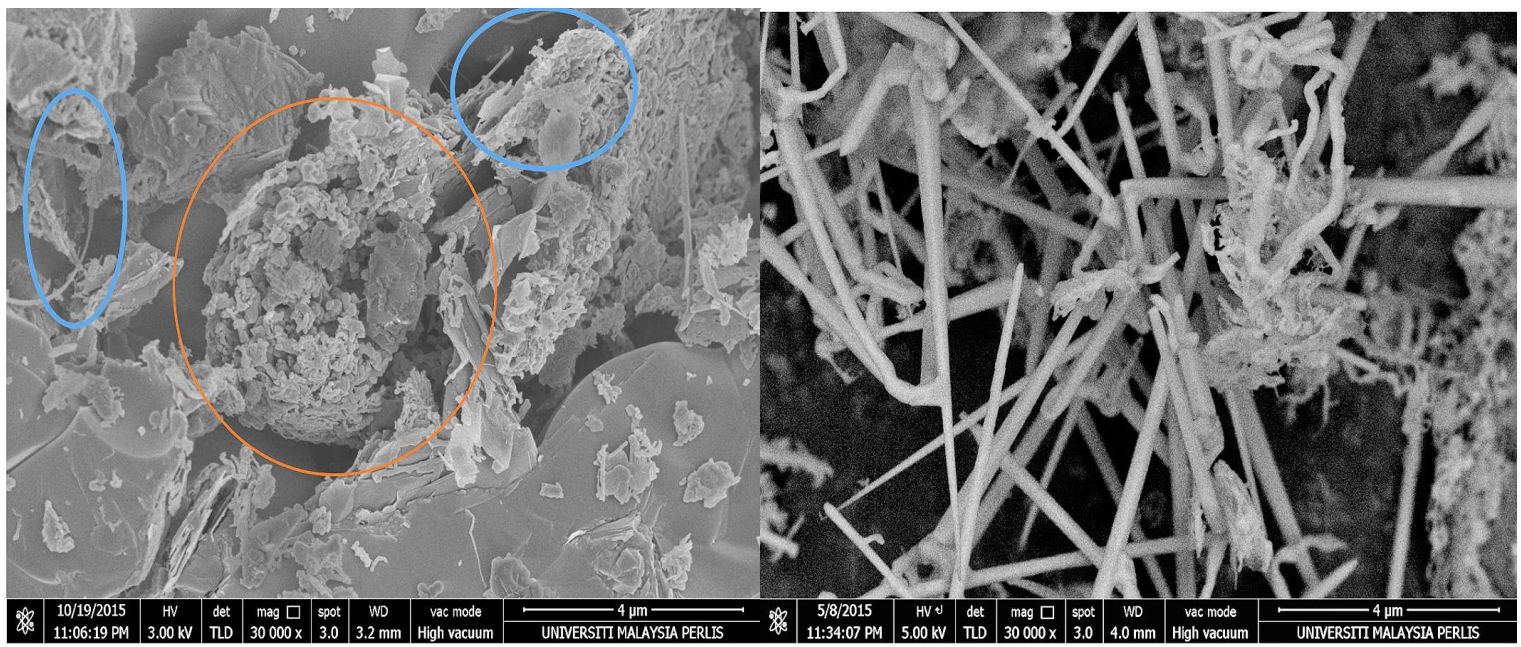

(a)

(b)

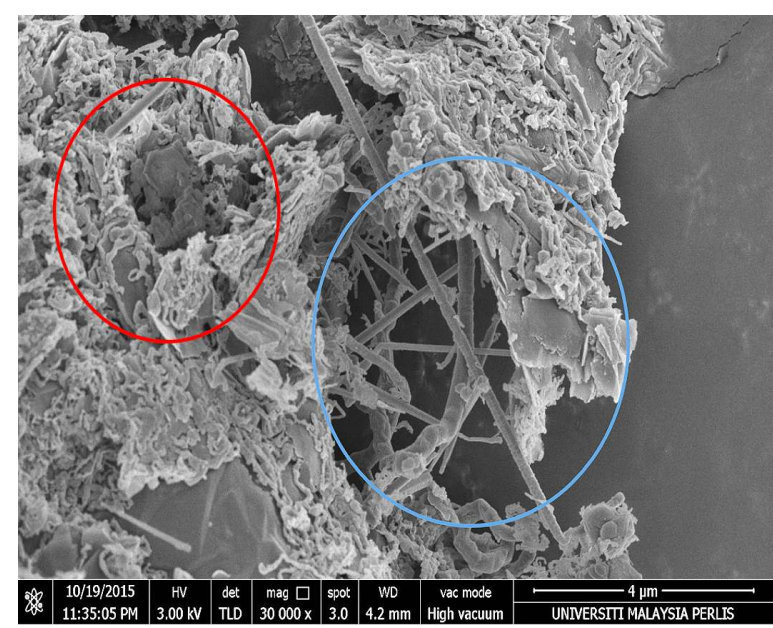

(c)

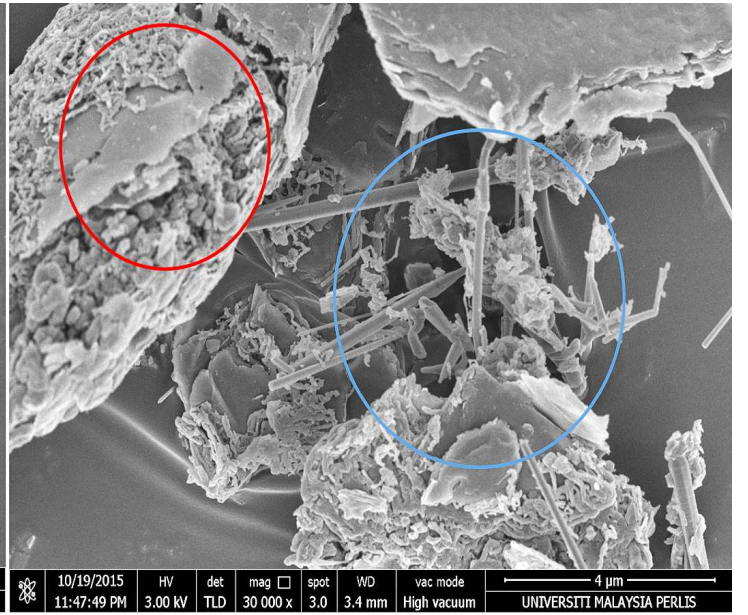

(d)

Fig. 2. FESEM images of the mixtures of graphite and silica in the ratio of (a) 1:1; (b) 1:3; (c) 1:5 and (d) $1: 7$ after subjecting to microwave heating. Orange circle: $\mathrm{SiO}_{2}$ unreacted, blue circle: $\mathrm{SiCNW}$ and red circle: unreacted graphite.

\subsection{XRD patterns characterization}

XRD patterns of SiCNWs synthesized from the mixtures of different ratios of $\mathrm{SiO}_{2}$ and graphite are shown in Fig. 3. In Fig. 3a, the peak corresponding to $\mathrm{SiO}_{2}$ at $2 \theta$ of $22.3^{\circ}$, associated with (1 $\left.\begin{array}{lll}1 & 0\end{array}\right)$ plane is noticed. The peak of $\beta$-SiC $\left(\begin{array}{lll}1 & 1 & 1\end{array}\right)$ with low intensity is observed at the $2 \theta$ values of $36^{\circ}$. This indicates that for the ratio $1: 1$ of $\mathrm{SiO}_{2}$ and graphite, insufficient amount of carbon caused the incomplete synthesis of SiCNWs. Small peak of SiC has appeared due to the side reaction of the formed $\mathrm{SiC}$ with the excess $\mathrm{SiO}_{2}$ forming $\mathrm{SiO}$ and $\mathrm{CO}$ gases, which led to the depletion of SiC phase [22]. This observation is in good consistency with the corresponding FESEM image in Fig. 2a, in which only small amount of SiCNWs was observed.

Fig. $3 \mathrm{~b}$ shows the XRD pattern of SiCNW synthesized from the mixture of $\mathrm{SiO}_{2}$ and graphite in the ratio of $1: 3$. Three peaks that correspond to B-SiC, with (1 11 1), ( $\left.\begin{array}{lll}2 & 2 & 0\end{array}\right)$ and (3 111 ) crystal planes of cubic $\beta$-SiC (JCPDS Card No. 074-2307) are observed at $2 \theta$ values of $36^{\circ}, 61^{\circ}$ and $72.5^{\circ}$. 
Ceballos-Mendivil et al. [23] also reported a similar observation that $\beta$-SiC has diffraction peaks at $2 \theta$ values of $35.6^{\circ}, 60^{\circ}$ and $71.8^{\circ}$ corresponding to

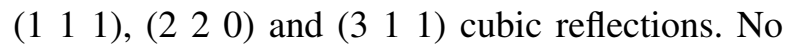
signal of either $\mathrm{SiO}_{2}$ or carbon is observed in this XRD pattern. It can be concluded that the mixture of $\mathrm{SiO}_{2}$ and graphite in the ratio 1:3 was converted completely to SiCNWs. This result is in good agreement with the FESEM images in Fig. 2b in which SiCNWs were observed without the traces of graphite or silica particles.

For SiCNWs synthesized from the mixture of $\mathrm{SiO}_{2}$ and graphite in the ratio of 1:5, as in Fig. 3c, the peaks corresponding to $\beta-\mathrm{SiC}$ appear at $2 \theta$ values of $36^{\circ}, 61^{\circ}$ and $72.5^{\circ}$, respectively. However, the relative intensities of these peaks are lower compared to those in Fig. 3b. A small peak of carbon phase observed also at $27^{\circ}$ corresponds to $\left(\begin{array}{lll}0 & 0 & 2\end{array}\right)$ plane of graphite. Generally, the presence of carbon phase is due to the excess of unreacted graphite. In consistency with FESEM image in Fig. 2c, the amount of SiCNWs formed from the mixture of $\mathrm{SiO}_{2}$ and graphite in the ratio of 1:5 decreased comparing to Fig. $2 b$ while irregular particles of unreacted graphite are observed. As displayed in Fig. 3d, the peaks are similar to that in Fig. $3 \mathrm{c}$ but the intensity of the peak of carbon phase at $2 \theta$ of $27^{\circ}$ has risen slightly; meanwhile the peak of $\beta$-SiC decreased slightly compared to Fig. 3c. It is in good consistency with Fig. $2 d$ but the amount of SiCNWs is lower compared to FESEM image of Fig. 2c. Changhong et al. [24] proposed that an excess of carbon in synthesis of $\mathrm{SiC}$ is advantageous due to preventing undesired agglomeration of $\mathrm{SiC}$ powder but too large amount of carbon may affect the amount of produced SiC.

\subsection{Energy-dispersive X-ray spec- troscopy (EDX)}

Fig. 4 shows the EDX spectra of the SiCNWs synthesized by microwave irradiation from mixtures in the ratios of 1:1, 1:3, 1:5 and 1:7. Fig. 4a shows the EDX pattern of SiCNWs synthesized from the mixture of silica and graphite with the ratio of 1:1. From the peaks, 3 elements were detected which are $\mathrm{Si}, \mathrm{C}$ and $\mathrm{O}$. O element

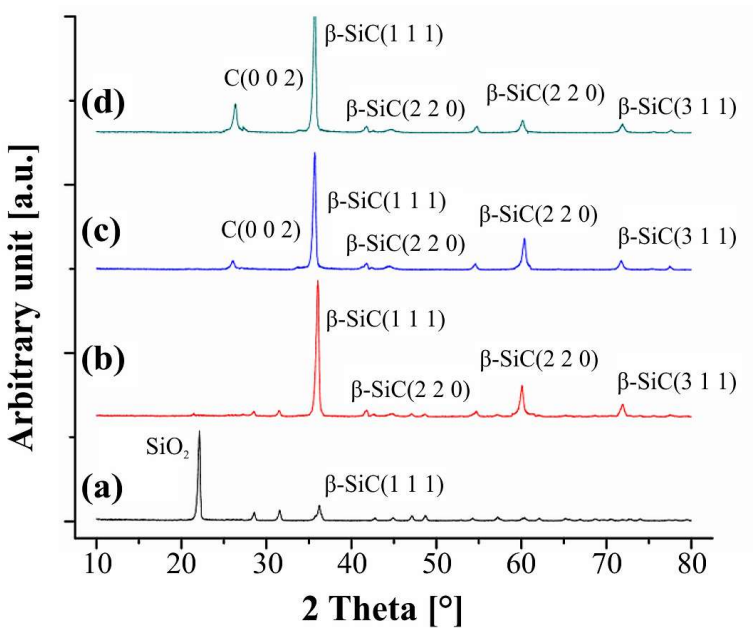

Fig. 3. XRD patterns of SiCNW synthesized by microwave heating of the mixtures of $\mathrm{SiO}_{2}$ and graphite in the ratios of (a) $1: 1$; (b) $1: 3$; (c) $1: 5$ and (d) $1: 7$.

corresponds to the presence of silica in the final product. This indicates that silica has not fully reacted in this process, which is in good consistency with the XRD result in Fig. 3 and FESEM image in Fig. 2a. Similar observation was also reported by Quah et al. [25] and they attributed the presence of $\mathrm{O}$ element in the EDX spectrum to the presence of unreacted $\mathrm{SiO}$ in the final product.

In the EDX spectrum of SiCNWs synthesized from the mixture with the ratio $1: 3$, peaks corresponding to $\mathrm{Si}$ and $\mathrm{C}$ elements were found. This indicates that the mixture of $\mathrm{SiO}_{2}$ and graphite in the ratio of 1:3 reacted completely to form SiCNWs.

As shown in Fig. 4c and Fig. 4d, the EDX spectra of SiCNWs synthesized from the mixture of $\mathrm{SiO}_{2}$ and graphite with the ratios of 1:5 and 1:7 consist of peaks corresponding to $\mathrm{C}$ and $\mathrm{Si}$, respectively. However, the intensity of EDX peak corresponding to $\mathrm{C}$ increased as the ratio of $\mathrm{C}$ to $\mathrm{SiO}_{2}$ in the mixture of raw materials increased. This is because of the presence of graphite that has not reacted with $\mathrm{SiO}_{2}$ during the microwave heating process due to insufficient amount of $\mathrm{SiO}_{2}$. This result is in good agreement with XRD patterns and SEM images of SiCNWs formed from the mixtures of $\mathrm{SiO}_{2}$ and graphite with the ratios of $1: 5$ and 1:7, respectively. 


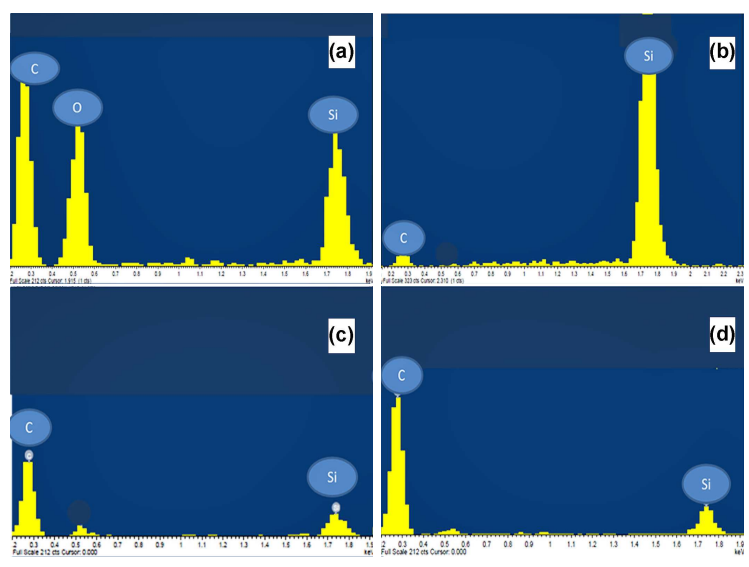

Fig. 4. EDX spectra of the SiCNWs synthesized by using microwave heating from the mixtures of $\mathrm{SiO}_{2}$ and graphite with the ratios of (a) $1: 1 ;$ (b) $1: 3$; (c) $1: 5$ and (d) $1: 7$.

\subsection{Fourier transform infrared spec- troscopy (FT-IR)}

FT-IR transmission spectra of SiCNWs synthesized from the mixtures with different ratios of graphite and $\mathrm{SiO}_{2}$ are shown in Fig. 5. From the graph, it can be concluded that the SiCNWs were successfully synthesized since the FT-IR peaks correspond to $\mathrm{Si}-\mathrm{C}$ stretching bond present at $1000 \mathrm{~cm}^{-1}$ to $800 \mathrm{~cm}^{-1}$ in all the FT-IR spectra of SiCNWs. From Fig. 5a, it can be observed that SICNWs synthesized from the mixture of $\mathrm{SiO}_{2}$ and graphite with the ratio of 1:1 has FT-IR peak corresponding to stretching bond of $\mathrm{Si}-\mathrm{O}$ bonding group at $1110 \mathrm{~cm}^{-1}$. The presence of this absorption band indicates the presence of $\mathrm{SiO}_{2}$. Similar absorption bands were reported by Zhao et al. [8] and Rajarao et al. [26]. Zhao et al. obtained such absorption peak at $1080 \mathrm{~cm}^{-1}$ and they suggested that this peak was associated with the $\mathrm{Si}-\mathrm{O}-\mathrm{Si}$ bond of mesoporous silica. Rajarao et al. also reported absorption band at $1045 \mathrm{~cm}^{-1}$ and this peak was attributed to $\mathrm{Si}-\mathrm{O}-\mathrm{Si}$ bond. This result indicated that some of the $\mathrm{SiO}_{2}$ was unreacted. This was due to the fact that the amount of available graphite was insufficient to react completely with $\mathrm{SiO}_{2}$ and thus caused some of $\mathrm{SiO}_{2}$ was left as residue.

For the FT-IR spectrum of SiCNWs formed from the mixture of graphite and $\mathrm{SiO}_{2}$ with 1:3

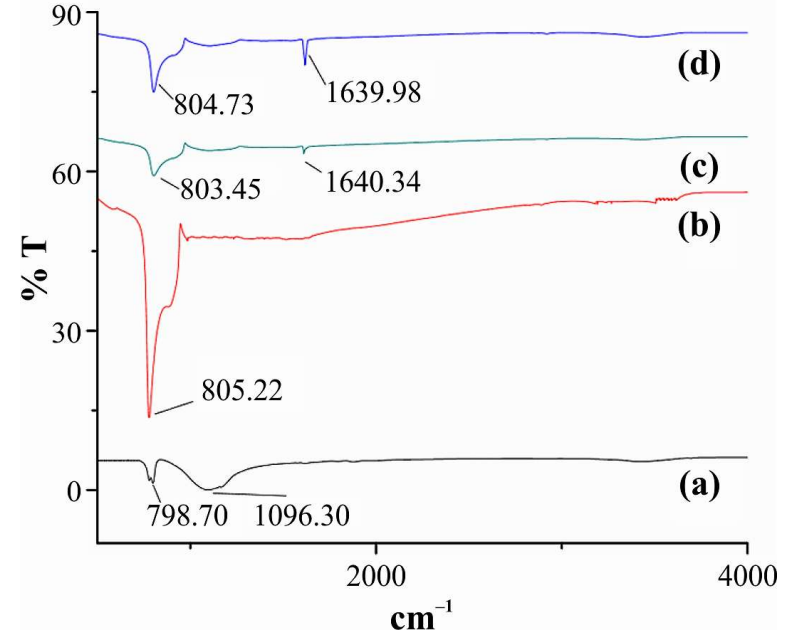

Fig. 5. FT-IR spectra of SiCNWs synthesized using the microwave heating from the blend of $\mathrm{SiO}_{2}$ and graphite in the ratio of (a) $1: 1$; (b) $1: 3$; (c) $1: 5$ and (d) 1:7.

ratio, the peak appearing at $805.22 \mathrm{~cm}^{-1}$ corresponds to the presence of $\mathrm{Si}-\mathrm{C}$ bond which indicates the successful synthesis of SiCNWs. Similar absorption peak was reported in the study of Rajarao et al. [26], which attributed absorption band at $805 \mathrm{~cm}^{-1}$ to $\mathrm{Si}-\mathrm{C}$ bond. This result is in good consistency with the XRD result of SiCNWs synthesized with the ratio 1:3 shown in Fig. 3b, which indicates the presence of only a single phase $\beta-\mathrm{SiC}$ and thus denotes complete conversion of graphite to SiCNWs. FT-IR spectra of SiCNWs were synthesized from a blend of $\mathrm{SiO}_{2}$ and graphite in the ratio of $1: 5$ and $1: 7$ as shown in Fig. 5c and Fig. $5 \mathrm{~d}$ revealing the presence of absorption peak corresponding to $\mathrm{C}=\mathrm{C}$ stretching bonds centered at $1640 \mathrm{~cm}^{-1}$. Absorption bands at $1630 \mathrm{~cm}^{-1}$ to $1640 \mathrm{~cm}^{-1}$ are also observed in Fig. 5c and Fig. 5d and these absorption bands are due to the presence of $\mathrm{C}=\mathrm{C}$ bonds of graphite [27]. The intensity of absorption band of $\mathrm{C}=\mathrm{C}$ is higher in Fig. $5 \mathrm{~d}$ comparing to Fig. $5 \mathrm{c}$ because the increased amount of graphite in the mixture of silica and graphite of 1:7 ratio compared to $1: 5$ ratio.

\subsection{Photoluminescence characterization}

PL spectra of SiCNWs synthesized from the mixtures with different $\mathrm{SiO}_{2}$ and graphite ratios are 
shown in Fig. 6. Fig. 6 shows the peaks of SiCNWs at $440 \mathrm{~nm}(2.8 \mathrm{eV})$ in all spectra. The peaks are obviously blue-shifted in comparison with the band gap of 3C-SiC $(2.39 \mathrm{eV})$. The blue shift of the PL peak of $3 \mathrm{C}-\mathrm{SiC}$ nanomaterials has been reported by several researchers [28-31]. For example, the peak at $418 \mathrm{~nm}$ has been detected in $3 \mathrm{C}-\mathrm{SiC}$ nanobelts by $\mathrm{Wu}$ et al. [32]. They proposed that the origin of this peak depends on the nanostructure, morphology and size of 3C-SiC samples. The collective influence of size confinement effect and defects lead to the blue-shift of the peak. Thus, the peak emission that appeared at around $440 \mathrm{~nm}$ may be related to size confinement effect and defects.

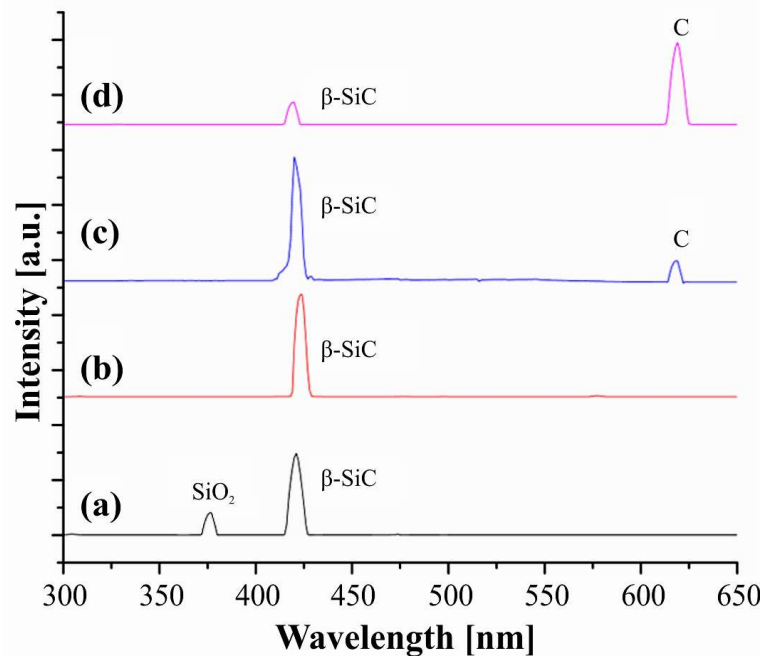

Fig. 6. PL spectrum of SiCNWs synthesized using the microwave heating from the mixture of $\mathrm{SiO}_{2}$ and graphite in the ratio of (a) $1: 1$; (b) $1: 3$; (c) $1: 5$ and (d) 1:7.

Fig. 6a displays PL spectrum of SiCNWs synthesized from the blend of $\mathrm{SiO}_{2}$ and graphite in the ratio of 1:1 which shows the presence of PL peak attributed to oxygen discrepancy in $\mathrm{SiO}_{2}$ at the wavelength $\sim 380 \mathrm{~nm}$ corresponding to band gap of $3.2 \mathrm{eV}$. This PL spectrum is in good consistency with the XRD result of SiCNWs synthesized from the blend of $\mathrm{SiO}_{2}$ and graphite in the ratio of $1: 1$, which shows the presence of XRD peak corresponding to $\mathrm{SiO}_{2}$. Nandanwar et al. [33] studied $\mathrm{SiO}_{2}$ nanoparticles that also indicated PL spectrum of pure $\mathrm{SiO}_{2}$ with similar result at $381.8 \mathrm{~nm}$.
Fig. 6b shows that in the PL spectrum of SiCNWs formed from the mixture with the ratio of $\mathrm{SiO}_{2}$ and graphite of 1:3, only one peak appeared at $425 \mathrm{~nm}$. The peak corresponds to $\beta-\mathrm{SiC}$. This indicates that only $\mathrm{SiC}$ is present in the SiCNWs synthesized from this mixture. This result is in good consistency with the XRD result in Fig. 3b showing that graphite and $\mathrm{SiO}_{2}$ reacted completely forming single phase SiCNWs.

For the SiCNWs synthesized from the blend of $\mathrm{SiO}_{2}$ and graphite with the ratios of 1:5 and 1:7 as shown in Fig. $6 \mathrm{c}$ and d, it can be seen that the peaks corresponding to $\beta-\mathrm{SiC}$ and carbon are observed at the wavelength of $420 \mathrm{~nm}$ and $620 \mathrm{~nm}$ with band gaps of $2.9 \mathrm{eV}$ and $2.0 \mathrm{eV}$, respectively. This indicates that there is unreacted graphite in the SiCNWs [34].

\subsection{Thermal gravimetric analysis of SiCNWs}

Thermal gravimetric analysis (TGA) results of SiCNWs synthesized from the mixtures with different ratios of silica and graphite are presented in Fig. 7. TGA was conducted to evaluate the quality of SiCNWs indirectly. For SiCNWs synthesized from the mixture with 1:1 ratio (Fig. 7b), the weight loss started at $700{ }^{\circ} \mathrm{C}$ with a total of $8 \%$ weight loss. The weight loss of SiCNWs can be attributed to the oxidation of unreacted carbon from the synthesis of SiCNWs. Corriu et al. [35] proposed that the weight loss which occurred in the range of $450{ }^{\circ} \mathrm{C}$ to $750{ }^{\circ} \mathrm{C}$ was assigned to the air oxidation of the carbon. Smaller weight loss occurred for SiCNWs formed from the mixture with 1:3 ratio as compared to SiCNWs formed from other mixtures with just $6 \%$ of weight loss. This weight loss may be attributed to the oxidation of carbon. Lower weight loss of SiCNWs synthesized from the mixture of 1:3 ratio compared to that synthesized from the mixture of $1: 1$ ratio is due to the absence of carbon, because graphite has been fully converted into SiCNWs. This result is in good agreement with XRD result in Fig. $3 b$ where the amount of carbon in $\mathrm{SiCNW}$ formed from the mixture with the ratio 1:3 was too low to be detected. This high resistance toward oxidation shown by the SiCNWs made from 
1:3 ratio of silica and graphite is attributed to the formation of pure SiCNWs.

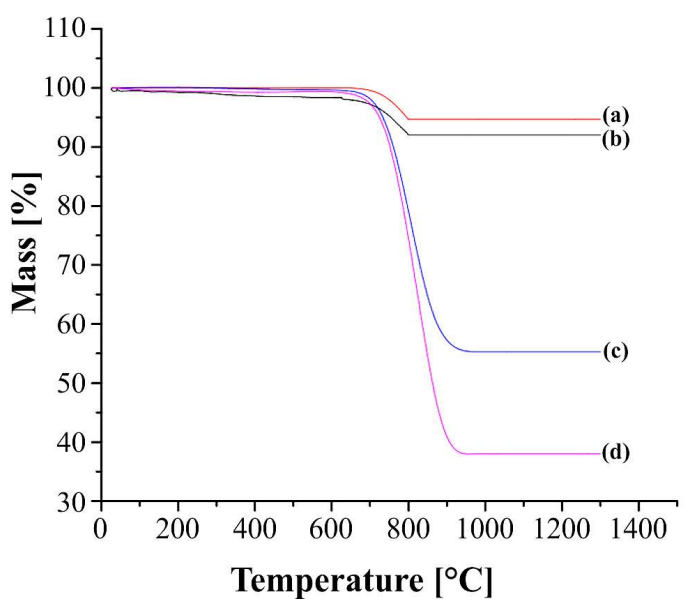

Fig. 7. TGA curves obtained by microwave heating of SiCNWs synthesized from the mixtures of $\mathrm{SiO} 2$ and graphite in the ratios of (a) 1:3 (weight loss $6 \%$ ); (b) 1:1 (weight loss $8 \%$ ); (c) 1:5 (weight loss $45 \%$ ); (d) 1:7 (weight loss $65 \%$ ).

Fig. 7c shows that for SiCNWs synthesized from the mixture of 1:5 ratio, $45 \%$ weight loss is observed from $700{ }^{\circ} \mathrm{C}$ to $950{ }^{\circ} \mathrm{C}$, while for SiCNWs synthesized from the mixture of $1: 7$ ratio in Fig. 7d, $65 \%$ weight loss is observed from $700{ }^{\circ} \mathrm{C}$ to $950{ }^{\circ} \mathrm{C}$. The increase in weight loss is due to the oxidation of carbon in SiCNWs formed from the mixtures with 1:5 and 1:7 ratios. The weight loss of SiCNWs synthesized from mixture of 1:7 ratio is higher compared to SiCNWs synthesized from mixture with 1:5 ratio, due to the excess of carbon in the remaining SiCNWs. The results are in good consistency with the XRD result from Fig. 3 that indicated the presence of higher amount of excess carbon for SiCNWs synthesized from the mixture with 1:7 ratio. This result demonstrates that SiCNWs produced from the mixture with the ratio of silica and graphite 1:3 has the highest thermal stability.

\subsection{Growth of SiCNWs by microwave heating}

Interaction of dielectric materials such as graphite with microwaves leads to what is generally described as dielectric heating. The interaction of charged particles in some materials with the electric field component of electromagnetic radiation causes these materials to heat up [36]. Graphite is known as carbon-based material that contains charged particles which are free to move in a delimited region of the material $[36,39]$. When electromagnetic field is applied to a material such as graphite, current being in phase with the electromagnetic field is induced. The electrons from the carbon material that cannot couple to the changes of phase in the electric field cause energy to dissipate in the form of heat. Fig. 8 shows the mechanism of dielectric heating that is based on the motion of electrons from carbon material to generate heat. Unlike liquid that has freely-rotatable dipole, carbon generates heat from the motion of electrons through Joule heating within the grains. This reaction is called Maxwell-Wagner effect that is quite different from the reaction of electromagnetic wave to a liquid such as water that heats up due to vibration of molecules [36, 37]. Calorimetric study of microwave absorption proposed by $\mathrm{He}$ et al. [38] showed that silica as an inorganic material almost cannot react to microwaves and the reaction is not as effective as in carbon based materials. Since the quartz material is not sensitive to microwave, we suggest that the heat from graphite (carbon based material) is transferred to silica via external radiant energy, such as conduction, convection and radiation, to assist the heating of silica. The homogeneous blend of silica and graphite therefore significantly affects the uniformity of temperature increase for both materials.

SiCNWs grow due to the carbothermal reduction between carbon and silica. The reduction of silica by carbon takes place through the following overall reaction [4]:

$$
\mathrm{SiO}_{2}(\mathrm{~s})+3 \mathrm{C}(\mathrm{s})=\mathrm{SiC}(\mathrm{s})+2 \mathrm{CO}(\mathrm{g})
$$

The reactions between silica and graphite are believed to occur in multiple steps before the production of SiCNWs. First reaction is the carbothermal reduction of silica and graphite to form $\mathrm{SiO}$ and $\mathrm{CO}$ gases by the following reaction:

$$
\mathrm{SiO}_{2}(\mathrm{~s})+\mathrm{C}(\mathrm{s}) \rightarrow \mathrm{SiO}(\mathrm{g})+\mathrm{CO}(\mathrm{g})
$$




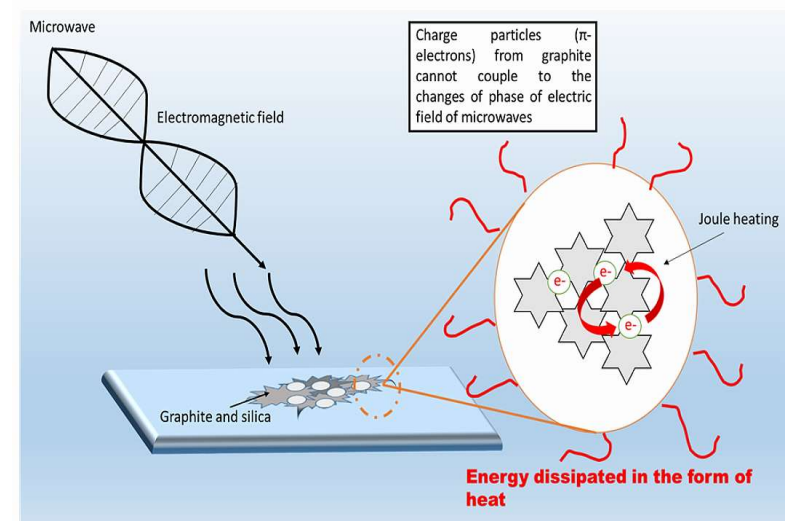

Fig. 8. Interaction of microwave with graphite leading to dielectric heating of graphite.

The vapor-solid (VS) mechanism was suggested to explain the formation of SiCNWs. In reaction $4, \mathrm{SiO}$ reacts with $\mathrm{C}$ to produce $\mathrm{SiC}$ nucleus as follow:

$$
\mathrm{SiO}(\mathrm{g})+2 \mathrm{C}(\mathrm{s}) \rightarrow \mathrm{SiC}(\mathrm{s})+\mathrm{CO}(\mathrm{g})
$$

Cetinkaya et al. [22] stated that Si-containing vapors such as $\mathrm{Si}$ or $\mathrm{SiO}$ vapors react with $\mathrm{CO}$ gas or $\mathrm{C}$ solid to form $\mathrm{SiC}$ nuclei for the formation of SiCNWs through VS mechanism. SiC particles from reaction 4 are believed to serve as nucleation sites for VS mechanism:

$$
\mathrm{SiO}(\mathrm{g})+3 \mathrm{CO}(\mathrm{g}) \rightarrow \mathrm{SiCNWs}(\mathrm{s})+2 \mathrm{CO}_{2}(\mathrm{~g})
$$

From reaction 5, the VS mechanism occurs when $\mathrm{SiO}$ vapor and $\mathrm{CO}$ vapor have deposited at the tip of nuclei that formed from the previous reaction. The whisker grows along the direction of the least stable plane and forms SiCNWs as shown in Fig. 9. Fu et al. [39] proposed that the nanowire growth might be attributed to the direct reaction between $\mathrm{Si}$ and carbon atoms based on the adsorption and diffusion processes from preferential tips of the crystal nuclei. Fig. 10 shows the overall reaction from carbon atom and $\mathrm{SiO}$ vapor to form a nucleation site. Then, in the reaction between $\mathrm{SiO}$ and $\mathrm{CO}$ vapors first the whiskers, and finally SiCNWs are formed.

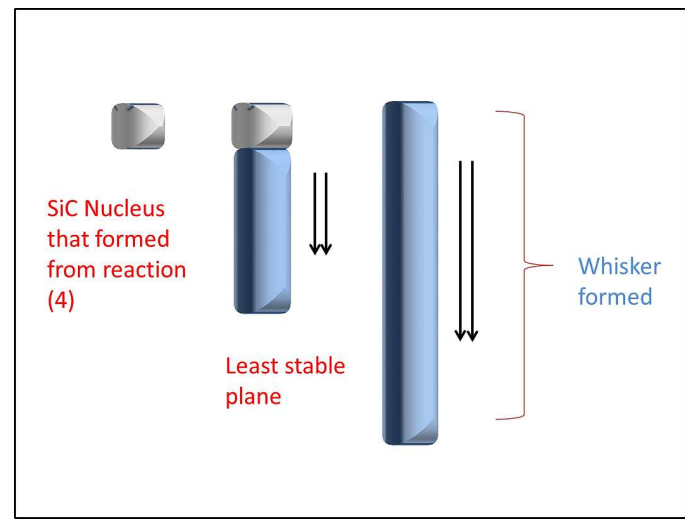

Fig. 9. Formation of a whisker by VS mechanism.

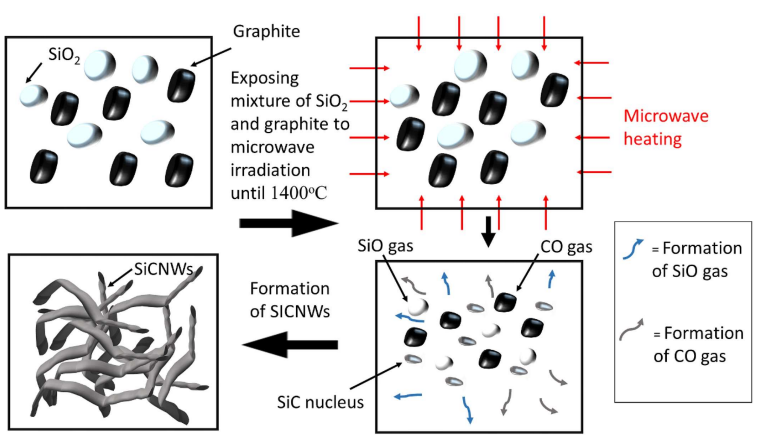

Fig. 10. Schematic of SiCNWs growth from reaction of $\mathrm{SiO}$ and $\mathrm{CO}$ vapor.

\section{Conclusions}

$\mathrm{SiC}$ nanowhiskers have been successfully synthesized through the microwave heating of a mixture of $\mathrm{SiO}_{2}$ and graphite. The experiment was studied by using different ratios of $\mathrm{SiO}_{2}$ /graphite. SiCNWs were characterized by X-ray diffraction (XRD), field emission scanning electron microscopy (FESEM), energy dispersive X-ray spectroscopy (EDX), photoluminescence spectroscopy (PL), Fourier transform infrared (FT-IR) and thermo-gravimetric analysis (TGA). The blend of $\mathrm{SiO}_{2}$ and graphite with the ratio 1:3 was found to be ideal for the synthesis of SiCNWs because of complete reaction between $\mathrm{SiO}_{2}$ and graphite resulting in the formation of single phase $\beta$-SiC nanowhiskers without any residue of $\mathrm{SiO}_{2}$ or graphite. Blends of $\mathrm{SiO}_{2}$ and graphite with the ratios 1:5 and 1:7 had the traces of unreacted graphite indicating that the conversion of graphite to 
SiCNWs was incomplete while unreacted $\mathrm{SiO}_{2}$ was detected in the mixture with 1:1 ratio of graphite and $\mathrm{SiO}_{2}$. This study describes a potential way of preparation of nanostructures (nanowhiskers) using graphite and silica by microwave heating, which can be a synthesis model for other materials blending.

\section{Acknowledgements}

The authors are grateful to the Department of Higher Education, Ministry of Higher Education, Malaysia, for funding this research through the Fundamental Research Grant Scheme (FRGS) with the Grant Number 9003-00441. The authors also would like to acknowledge all the team members in the Institute of NanoElectronic Engineering (INEE), Universiti Malaysia Perlis (UniMAP) for their guidance and help.

\section{References}

[1] Lutsenko V.G., Acta Mater, 56 (11) (2008), 2450.

[2] Najafi A., Golestani-Fard F., Rezaie H.R., EhSANi N., J. Sol-Gel Sci. Technol., 59 (2) (2011), 205.

[3] Martin H.-P., Ecke R., Müller E., J. Eur. Ceram. Soc., 18 (12) (1998), 1737.

[4] Li B., Zhang C., Hu H., CaO Y., Qi G., LiU R., J. Mater. Eng. Perform., 16 (6) (2007), 775.

[5] Prabhakaran P.V., SReejith K.J., SwamiNATHAN B., PACKIRISAMY S., NINAN K.N., J. Mater. Sci., 44 (2) (2008), 528.

[6] Naja A., Fard F.G., Rezaie H.R., Ehsani N., Powder Technol., 219 (2012), 202.

[7] Deng S.Z., Li Z.B., WANG W.L., Xu N.S., Zhou J., Zheng X.G., Xu H.T., Chen J., She J.C., Appl. Phys. Lett., 89 (2) (2006), 23.

[8] Zhao B., Zhang H., TaO H., Tan Z., Jiao Z., Wu M., Mater. Lett., 65 (11) (2011), 1552.

[9] Zhu J., Wu H., Chen H.T., Wu X.L., Xiong X., Phys. Lett. A, 373 (18) (2009), 1697.

[10] Li B., Song Y.-C., Zhang C.-R., Yu J.-S., Ceram. Int., 40 (8) (2014) 12613.

[11] Raman V., Bahl O.P., Dhawan U., J. Mater. Sci., 30 (1995), 2686.

[12] Lin M., Loh K.P., Boothroyd C., Du A.Y., Appl. Phys. Lett., 85 (22) (2004), 5388.

[13] Ho G.W., Wong A.S.W., Kang D.-J., Welland M.E., Nanotechnology, 15 (8) (2004), 996.

[14] Agrawal D.K., Curr. Opin. Solid St. M., 3 (5) (1998), 480.

[15] Mingos D.M.P., Adv. Mater., 5 (11) (1993), 857,

[16] Oghbaei M., Mirzaee O., J. Alloy. Compd., 494 (1) (2010), 175.

[17] Rao K.J., Vaidhyanathan B., Ganguli M., RAMAKRISHNAN P.A., Chem. Mater., 11 (1999), 882.

[18] Koguchi M., KaKibayashi H., Yazawa M., Hiruma K., Katsuyama T., Jpn. J. Appl. Phys., 31 (7R) (1992), 2061.
[19] Tong L., Reddy R.G., Mater. Res. Bull., 41 (12) (2006), 2303.

[20] Zhu W.Z., Yan M., Mater. Chem. Phys., 55 (1) (1998), 68.

[21] Biernacki J.J., Wotzak G.P., J. Am. Ceram. Soc., 72 (1) (1989), 122.

[22] Cetinkaya S., Eroglu S., J. Eur. Ceram. Soc., 31 (5) 2011, 869.

[23] Ceballos-Mendivil L.G., Cabanillas-López R.E., TÁNORI-Córdova J.C., MUrRIETAYescas R., PÉrez-Rábago C.A., VillafánVidAles H.I., ARANCIBIA-Bulnes C.A., EstradA C.A., Sol. Energy, 116 (2015), 238.

[24] Changhong D., Xianpeng Z., Jinsong Z., Yonguin Y., Lihua C., Fei X., J. Mater. Sci., 32 (9) (1997) 2469.

[25] Quah H.-J., Cheong K.Y., Lockman Z., J. Alloy. Compd., 475 (1) (2009), 565.

[26] Rajarao R., Ferreira R., SAdi S.H.F., Khanna R., Sahajwalla V., Mater. Lett., 120 (2014) 65.

[27] J. COATES, Interpretation of Infrared Spectra, A Practical Approach, in: MEYERS R.A. (Ed.), Encyclopedia of Analytical Chemistry, John Wiley \& Sons, Inc., Chichester, 2000, p. 10815.

[28] ChiU S.-C., Li Y.-Y., J. Cryst. Growth, 311 (4) (2009), 1036.

[29] Li Y., Chen C., Li J.-T., YAng Y., Lin Z.-M., Nanoscale Res. Lett., 6 (1) (2011), 454.

[30] Zhu J., Wu D.Y., Chen H., Xiong X., Chen X.B., Micro Nano Lett., 7 (9) (2012), 974.

[31] Arredondo Y.V.F., Cerámica y vidrio, 279 (c) (1969), 279.

[32] Wu R., Wu L., Yang G., Pan Y., Chen J., Zhai R., Lin J., J. Phys. D. Appl. Phys., 40 (12) (2007), 3697.

[33] Nandanwar R., Purnima S., Fozia Z., Am. Chem. Sci. J., 5 (1) (2015), 1.

[34] InIEwski K., Morris J.E. (Ed.), Graphene, Carbon Nanotubes, and Nanostructures: Techniques and Applications, CRC Press, Taylor \& Francis Group, Boca Raton - London - New York, 2013.

[35] Corriu R.J.P., Gerbier P., Guérin C., Henner B., J. Mater. Chem., 10 (9) (2000), 2173.

[36] Kim T., LeE J., LeE K.-H., Carbon Lett., 15 (1) (2014), 15.

[37] Menéndez J.A., Rahman R.A., Arenillas B.F., FERnández L.Z.Y., CAlvo E.G., Bermúdez J.M., Fuel Process. Technol., 91 (1) (2010), 1.

[38] He C.L., Chen Y.Q., J Microw. Power Electromagn. Energy, 47 (4) (2013), 251.

[39] Fu Q.-G., Li H.-J., SHi X.-H., Li K.-Z., WEI J., HU Z.-B., Mater. Chem. Phys., 100 (1) (2006), 108. 